\title{
Post-Operative Echocardiographic Changes between two Groups of CABG Patients: A Study in the National Institute of Cardiovascular Disease (NICVD)
}

\author{
Kazi Zahidul Hoque $^{1 *}$, Masumul Gani Chowdhury ${ }^{2}$, Md. Ashraful Islam ${ }^{3}$, Makbul Hossain ${ }^{4}$, \\ Md. Mostafizur Rahman ${ }^{5}$, Faizun Nessa Akther ${ }^{6}$, Lieutenant Colone ${ }^{1}$, Md. Zakir Hossain ${ }^{7}$ \\ ${ }^{I}$ Assistant Professor (Consultant) \& Unit Chief, Pediatric Cardiac Surgery Unit 2, Dhaka Shishu (Children) \\ Hospital \& Institute of Child Health, Consultant, Metropolitan Medical Center, Dhaka, Bangladesh \\ ${ }^{2}$ Assistant Professor \& Unit Chief, Pediatric Cardiac Surgery Unit 3, Dhaka Shishu (Children) Hospital \& \\ Institute of Child Health, Dhaka, Bangladesh \\ ${ }^{3}$ Junior Consultant, Department of Cardiac Surgery, Square Hospital Ltd, Dhaka, Bangladesh \\ ${ }^{4}$ Associate Professor, Pediatric Cardiac Anesthesiology, Dhaka Shishu (Children) Hospital \& Institute of Child \\ Health, Dhaka, Bangladesh \\ ${ }^{5}$ Professor, Cardiac Surgery, Bangabandhu Sheik Mujib Medical University, Dhaka, Bangladesh \\ ${ }^{6}$ Registrar, Department of Gynecology \& Obstetrics, Bangladesh Specialized Hospital, Dhaka, Bangladesh \\ ${ }^{7}$ Associate Professor and Head of the Department of Otolaryngology and Head-Neck Surgery, CMB, BAF, \\ Dhaka cantonment, Dhaka 1206, Bangladesh
}

*Corresponding Author: Kazi Zahidul Hoque, Assistant Professor (Consultant) \& Unit Chief, Pediatric Cardiac Surgery Unit 2, Dhaka Shishu (Children) Hospital \& Institute of Child Health, Consultant, Metropolitan Medical Center, Dhaka, Bangladesh, E-mail:kzhoque72@yahoo.com

\begin{abstract}
Introduction: Coronary artery bypass surgery both conventional method (Conventional Coronary Artery Bypass surgery-CCAB) and off-pump method (Off-pump Coronary Artery Bypass Surgery-OPCAB) are frequently performed operation especially in our National Institute of Cardiovascular Disease and Hospital.
\end{abstract}

Objective: To find out the Post-operative echocardiographic changes in between two groups of CABG patients.

Patients and Methods: The study was conducted in the department of Cardiovascular Surgery, National Institute of Cardiovascular Disease (NICVD), Dhaka, Bangladesh. AProspective Non-randomized clinical studyFrom January 2006 to November 2007. Study population the patients with ischemic heart disease with left main coronary artery disease who underwent off pump and on pump CABG in NICVD. Non probability sampling technique. Total Sample Size number of selected patients was sixty. Thirty patients in each group.

Observation and Results: Among 60 patient the mean age group I was $51.5 \pm 7.3$ and that of group II patient was $54.7 \pm 6$ years. The mean age difference was not statistically significant $(p>0.05)$ in unpaired t test. Among 60 patients 52 patients were male and 8 patients were female the percentages were $83.3 \%$ and $16.7 \%$ in group I and $90.0 \%$ and $10.0 \%$ in group II respectively. Male female ratio is 6.5:1. The male female differences was not statistically significant $(p>0.05)$ in chi square test.

Summary and Conclusion: In our institution more than a decade has almost over since the start of OPCAB surgery with gradual success and integrity. . This study is the pilot study and it never studied in any place before in Bangladesh. My effort in this study was to establish the safety and effectiveness of off pump coronary artery bypass surgery in left main coronary artery disease.

Keywords: $C C A B, O P C A B$, Risk factor, $C A B G$ patients.

\section{INTRODUCTION}

Coronary artery bypass surgery both conventional method (Conventional Coronary Artery Bypass surgery-CCAB) and off-pump method (Off-pump Coronary Artery Bypass Surgery-OPCAB) are frequently performed operation especially in our National Institute of Cardiovascular Disease and Hospital. So far there are several other modern techniques 
regarding coronary artery bypass surgery are emerging throughout the world, our aim is to practice those side by side with the developed world even within our limited resources and keep ourselves up to date. We should always consider the greater benefit of our patients in respect of economy, time and quick postoperative recovery. Surgical revascularization for atherosclerotic heart disease is one of the great success stories in medicine. Relief of angina after revascularization, improvement in exercise tolerance, and the realization of survival benefit have attended the operation since the early stages of development. The evolution of coronary surgery is a story of focused thought, dedication, courage, collaboration and serendipity [1]. Coronary Artery Bypass Graft (CABG) surgery is well-established treatment modalities in patients with Coronary Artery Diseases (CAD) refractory to medical therapy or when intervention cardiologic procedures (PTCI) are not feasible [2]. Surgical attempts at increasing blood flow to the ischemic myocardium originated a century ago when Alexis Carrel anastomosed a carotid artery segment between the descending aorta and the left coronary artery.

Three decades later, Arthur Vineberg started implanting the left internal thoracic artery into the anterior myocardial territory of patients with CAD in order to increase arterial inflow and relieve angina with some experiencing prolonged symptomatic improvement [3, 4, and 5].

Coronary surgery moved into modern era in the 1950s. It is not entirely clear to whom credit should be given for the first coronary bypass. The first direct surgical approach to the coronary circulation was likely performed by William Mustard in 1953 in Toronto, who used a carotid to coronary bypass though the patient did not survive the operation [1].

The first reported successful CABG operation took place in 1964 in Leningrad, where Kolesov grafted LIMA to the left anterior descending artery without CBP. Depending on referral patterns, approximately $40-50 \%$ of patients who undergo coronary angiography have significant involvement of all three coronary arteries i.e. RCA, LAD and LCX, however 5-10\% have stenosis of the left main stem. Approximately $5 \%$ of patients have diffuse disease of one or more major coronary branches [3, 4, and 5]. Incidence of coronary artery disease is increased in Bangladesh was reported as $11 \%$ which was third position after Rheumatic and Heart disease. The ratio of coronary artery disease between male and female is 11:1. [6]. Another survey in Bangladesh Hossain found that out of 500 population, $4.6 \%$ had cardiovascular problem also carried out a survey among 7062 urban and rural people and observed that $2.92 \%$ suffered from some sort of heart disease' (Anayetullah \& Barman 1991).

One of the most hotly debated and polarizing issues in cardiac surgery has been whether coronary artery bypass surgery without the use of cardiopulmonary bypass and cardioplegia (Off pump CABG) is superior to that performed with the heart lung machine and the heart's being chemically arrested (Conventional CABG).Initial descriptive studies reported excellent to outstanding outcomes with OPCAB, with the suggestion in many studies of less use of resources, less blood loss, less morbidity and a shortened length of hospital stay [3,4,5]. In one study done in the NICVD, Bangladesh there is less postoperative bleeding, less need for blood transfusion, less ventilation time, less ICU stay, hospital stay and cost effectiveness on OPCAB surgery[6].

\section{JUSTIFICATION OF THE STUDY}

Coronary artery disease with left main stem stenosis is one of the incremental peri and postoperative risk factor for the patient undergoing CABG. As because this artery along with its two major braches supply a greater territory of myocardium, which endangers due to occlusion of this vessel. However we are encountering this system of artery involvement day by day and the incidence of its involvement has been increasing gradually among patients. In the past all patients with coronary artery disease were selected for on pump conventional CABG, but gradually surgeons choice switched over to off pump CABG due to its equivalent safety, efficacy and cost effectiveness considering the economy of our country. But still conventional method had been applied in case of left main disease till introduction of off pump CABG technique by one of our skilled surgeon in recent days.

The scientific study regarding introduction of off pump technique in IHD patients with left main stem stenosis has been a crying need since its introduction. For the evidence of this foolproof technique, prediction of better result and to establish it as a safe procedure, this study is performed to usher a new era regarding off 
pump CABG surgery in left main coronary artery disease.

\section{AIMS AND OBJECTIVES}

To find out the Post-operative echocardiography changes in between two groups of CABG patients

\section{REVIEW OF LITERATURE}

Historically Arthur Vineberg in 1946 started implanting LIMA to anterior myocardial territory with coronary artery disease in order to increase arterial inflow and relieve angina. Then Alexis Carrel anastomosed a carotid artery segment between the descending aorta and the left coronary artery in dog. Surgery on the coronary arteries was introduced clinically in 1958 by William Longmire, who reported on the use of endarterectomy in five patients operated without cardiopulmonary bypass. The first reported successful CABG operation took place in 1964 in Leningrand, where Kolesov grafted LIMA to the left anterior descending artery without CPB. The world's first CABG program started 3 years later in Cleveland, as Favaloro began to routinely use reversed saphenous veins for aorto-coronary grafting. The $\mathrm{CABG}$ procedure was then rapidly adopted and developed world wide $[3,4,5]$.

First clinical description of the left main coronary artery was made by Herrick in 1912; numerous studies have been shown that stenosis of the left main coronary artery is of critical prognostic importance. Observational studies of medically and surgically treated patients with LMCD support widely accepted belief that in general, coronary artery bypass graft surgery lessens symptoms and significantly prolongs survival [7, 8] studied eight hundred twenty three patient of bypass grafting for left main coronary artery. One hundred patients were re-vascularized without the use of cardiopulmonary bypass and compared with a 723 patients who underwent grafting with the aid of cardiopulmonary bypass. There was one death in off pump grafting as compared with a 30 day mortality of $4.7 \%$ in the on-pump group. In their study patients re-vascularized without cardiopulmonary bypass were significantly less likely to require post-operative inotropic support and transfusion, and it also revealed that cardiopulmonary bypass was an independent risk factor for mortality and came to a conclusion that coronary artery bypass grafting using off-pump techniques is safe and effective in left main coronary artery disease [8].
However it was already proved by the several studies that excessive peri-operative bleeding continues to complicate cardio-pulmonary bypass.' Since its successful clinical introduction by Gibbon 1953, use of cardiopulmonary bypass (CPB) has been the mainstay of cardiac surgery, facilitating a variety of complex procedures. Until recently, use of $\mathrm{CPB}$ was synonymous with coronary artery bypass surgery, a procedure of which it has been estimated over 8000,000 are performed annually worldwide' [9]. Independent of the cost, complexity, and high degree of technical support associated with CPB there has been steadily mounting evidence of some degree of end organ dysfunction associated with its usage. For a majority of the patients, these results in clinically undetectable sequel, for a significant minority, the result can be devastating [10]. In one study a total of 1988 of 10,647 patients who underwent a first isolated $\mathrm{CABG}$ at the Karolinska Hospital in Stockholm, Sweden, during 1970-1999 had significant left main coronary artery stenosis. The proportion of patients with LMCA stenosis of all CABG patients increased from $7 \%$ during the 1970 s to $26 \%$ in 1999 . During $1970-1984$ early mortality was $5.8 \%$ in patients with LMCA stenosis compared with $1.5 \%$ in patients without LMCA stenosis. The corresponding rates during 1995-1999 were $2.0 \%$ versus $2.2 \%$. Five-year survival in males was $88 \%$ after operations performed during 1994-1999 compared with $82 \%$ after CABG performed during 1970- 1984.

Five-year mortality, exclusive of early deaths, during 1970 - 1984 was higher in patients with LMCA stenosis than in those without. During 1970 - 1999 there was a decrease of early and five-year mortality in patients with LMCA stenosis after $\mathrm{CABG}$ despite increase of patient age and risk factors. The continuous decline of mortality during three decades most likely reflects improvement of the peri- and postoperative management of patients undergoing $\mathrm{CABG}$ during this period. (13 et al. 2006). In another study shows that 1,197 consecutive patients with critical left main stem stenosis $(>50 \%)$ underwent coronary surgery. Two hundred and fifty-nine $(21.6 \%)$ of these patients had off-pump coronary surgery, while 938 (78.4\%) received on-pump coronary surgery.

The requirement for inotropic support or a prolonged length of stay was significantly reduced after receiving off-pump coronary 
surgery. There was a trend to suggest that offpump patients had a lower incidence of stroke and chest infection. The adjusted freedom from death in off-pump patients at 2 years was $94.6 \%$ compared with $93.6 \%$ for on-pump patients [11].

\section{Patients And Methods}

Patients and Methods: The study was conducted in the department of Cardiovascular Surgery, National Institute of Cardiovascular Disease (NICVD), Dhaka, Bangladesh. A Prospective Non-randomized clinical study from January 2006 to November 2007. Study population the patients with ischemic heart disease with left main coronary artery disease who underwent off pump and on pump CABG in NICVD. Non probability sampling technique. Total Sample Size number of selected patients was sixty. Thirty patients in each group.

\subsection{Study Population and Sampling}

\subsubsection{Study Population}

Study population the patients with ischemic heart disease with left main coronary artery disease who underwent off pump and on pump CABG in NICVD

\subsubsection{Sampling Techniques}

Non probability sampling technique

\subsubsection{Sample Size}

Total number of selected patients was sixty. Thirty patients in each group.

\subsubsection{Population Group}

The total number of study population was divided into two groups based on the procedure of surgery. Each group has thirty populations.

Group I: CABG under cardiopulmonary bypass (On-Pump)

Group II: CABG without cardiopulmonary bypass (Off-Pump)

\subsection{Selection Criteria of the Patients}

\subsubsection{Inclusion Criteria}

Patients with LEFT MAIN Coronary Artery Disease undergoing CABG both off pump and on pump.

\subsubsection{Exclusion Criteria}

Patient having coronary artery disease other than left main disease

Patients having EF $<35 \%$

Patients with unstable angina, MI, and other significant ischemia events within $11 / 2$ month. Concomitant procedures including valvular operations. Has history of renal, respiratory or hepatic failure, stroke/TIA or coagulopathy.

\subsection{Patients Demographic Characteristics}

a) Age

b) $\operatorname{Sex}$

\subsubsection{CABG without Cardio-Pulmonary Bypass}

Perfection of technique and equipment for stabilizing the procedure the beating heart has resulted in an increased number of CABG performed without the use of $\mathrm{CPB}$ in our National Institute of Cardiovascular Disease and Hospital. Procedures involving placement of grafts to coronary arteries are performed through median sternotomy. At the same time segment of great saphenous vein is removed. Before the pericardium is opened the left Internal Mammary Artery is completely mobilized. Heparin is administered according to protocol. Then pericardiotomy done and stay suture are placed accordingly.

\subsubsection{Stabilizing and Positioning During $O P C A B$}

For stabilization purpose different modern stabilizers were used like 'Octopus' and 'Star Fish'. Octopus is a suction stabilizer for stabilizing the segment of coronary artery which is selected for placement of a graft, and Star Fish is a apical suction device for the purpose of elevating the heart for posterior exposure. After proper exposure and positioning the artery is incised and anastomosis to the appropriate conduit is performed. After distal graft proximal anastomosis is also performed with the aorta accordingly. After proper haemostasis ensured sternotomy closed and patient was taken to ICU with the support of mechanical ventilation [12].

\subsubsection{CABG with Cardio-Pulmonary Bypass}

After performing all protocol as mentioned above the median sternotomy done and at the same time a segment of great saphenous vein is harvested from the lower limb. Before the pericardium is opened the left IMA was completely mobilized and then divided and kept with a Bulldog Clamp. The pericardium then opened and stay sutures placed accordingly. The aortic cannula, aortic root cardioplegia cannula, double stage single cannula is inserted in the right atrium and commenced cardiopulmonary bypass.

When aorta is clamped then cardioplegia started and heart arrested in diastole. 
Then all anastomosis were performed in arrested heart according to the protocol. The patient was weaned from bypass and sent to ICU with the support of mechanical ventilation and other monitoring device [12].

\subsubsection{Follow up}

All patients had been followed up after one moth of discharge from hospital and subsequent two months in every one moth interval. In follow up period thorough clinical assessment, and Echocardiographic findings were checked to evaluate patient's post-operative cardiac function and surgical outcomes.

\subsubsection{Data Collection}

All relevant data for each participant where collected on redesigned Questionnaire (Appendix B)

\subsubsection{Statistical Analysis}

Data were collected by personal interview and hospital records. The collected data were compiled and a data file was constructed. This data were analyzed by unpaired student t test and Chi square test (yf) using SPSS (Statistical Program for Social Science). The analyzed data were presented by crossing of variables in the form of tables, and graph etc. A p value equal to or less than 0.05 was considered significant.

\section{OBSERVATION AND RESUltS}

Among 60 patients the mean age group I was $51.5 \pm 7.3$ and that of group II patient was $54.7 \pm$ 6 years. The mean age difference was not statistically significant $(p>0.05)$ in unpaired $t$ test.

Table1. Age distribution of the patients between the groups

\begin{tabular}{|c|c|c|c|c|c|}
\hline Age in years & Group I N & , & Group II N & $\%$ & P Value \\
\hline $45-50$ & 20 & 66.7 & 10 & 33.3 & \\
\hline $51-55$ & 4 & 13.3 & 12 & 40.0 & \\
\hline $56-60$ & 2 & 6.7 & 0 & 0.0 & \\
\hline $61-65$ & 0 & 0.0 & 8 & 26.7 & \\
\hline$>65$ & 4 & 13.3 & 0 & 0.0 & \\
\hline Mean age (yrs) & 51.5 & \pm 7.3 & 54.7 & \pm 6.0 & 0.063 \\
\hline \multicolumn{6}{|c|}{ 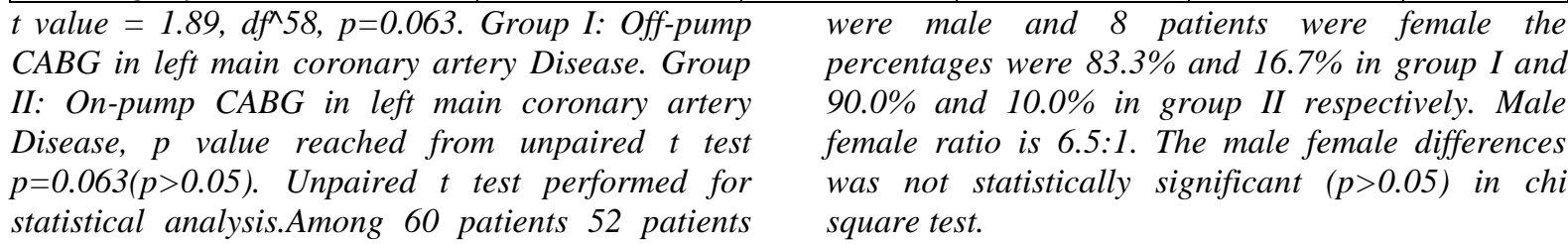 } \\
\hline
\end{tabular}

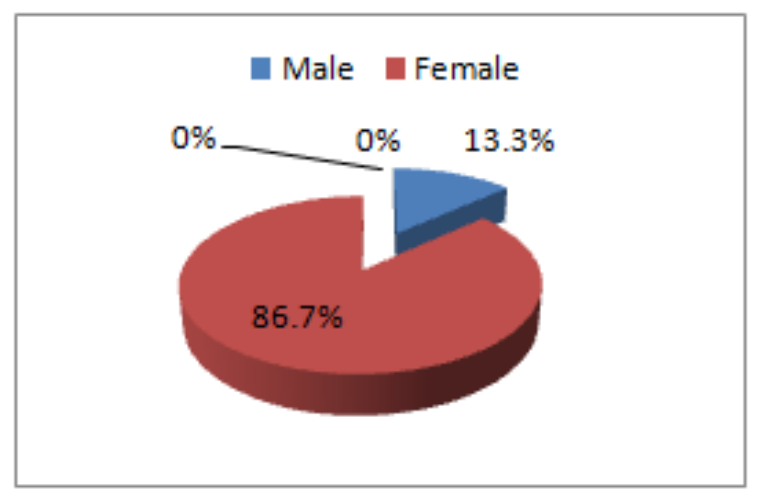

Fig1. Distribution of Sex

Chi value $=0.58, \mathrm{df}=1, \mathrm{p}=0.353$ Male female ratio: 6.5:1. Group I: Off-pump CABG in left main coronary artery Disease. Group II: Onpump CABG in left main coronary artery Disease, $p$ value reached from chi square test $\mathrm{p}=0.353(\mathrm{p}>0.05)$. Chi square test is performed for statistical analysis.Following table depicts the post-operative echocardiographic changes in between two groups of CABG patients in three successive months. These are LVIDd, LVIDs,
$\mathrm{EF}$ and wall motion abnormality. Unpaired $\mathrm{t}$ test is performed for LVIDd, LVIds, EF value and Chi square test is performed for wall motion abnormality value. No statistically significant (p>.\#5) difference is found among two groups in successive three months which means either off pump or on pump technique of CABG do not cause any effect on LVIDd, LVIDs, EF and wall motion abnormality change post operatively in CABG patients. 
Post-Operative Echocardiographic Changes between two Groups of CABG Patients: A Study in the National Institute of Cardiovascular Disease (NICVD)

Table2. Changes in Echocardigraphic variable between groups

\begin{tabular}{|c|c|c|c|c|c|}
\hline Variables & Group I & Group II & $\begin{array}{l}\text { t/chi } \\
\text { value }\end{array}$ & df & P-value \\
\hline \multicolumn{6}{|l|}{1 month } \\
\hline LVIDd (mm) & $47.5 \pm 1.1$ & $47.1 \pm 1.7$ & 1.08 & 58 & ${ }^{\mathrm{a}} 0.319^{\mathrm{ns}}$ \\
\hline LIVDs (mm) & $30.3 \pm 1.4$ & $31.1 \pm 1.8$ & 1.92 & 58 & ${ }^{\mathrm{a}} 0.061^{\mathrm{ns}}$ \\
\hline LVEF $(\%)$ & $59 \pm 2$ & $58 \pm 2$ & 1.94 & 58 & ${ }^{\mathrm{a}} 0.058^{\mathrm{s}}$ \\
\hline Wall motion abnormality & $2(6.7 \%)$ & $3(10.2 \%)$ & 0.22 & 1 & ${ }^{\mathrm{a}} 0.500^{\mathrm{ns}}$ \\
\hline 2 month & & & & & ${ }^{\mathrm{a}} 0.379^{\mathrm{ns}}$ \\
\hline LVIDd (mm) & $47.2 \pm 1.2$ & $46.9 \pm 1.4$ & 0.89 & 58 & ${ }^{\mathrm{a}} 0.080^{\mathrm{ns}}$ \\
\hline LIVDs (mm) & $30.1 \pm 1.3$ & $30.8 \pm 1.7$ & 1.79 & 58 & ${ }^{\mathrm{a}} 0.058^{\mathrm{ns}}$ \\
\hline $\operatorname{LVEF}(\%)$ & $60 \pm 2$ & $59 \pm 2$ & 1.94 & 58 & ${ }^{\mathrm{a}} 0.500^{\mathrm{ns}}$ \\
\hline Wall motion abnormality & $1(3.3 \%)$ & $2(6.7 \%)$ & 0.35 & 1 & \\
\hline \multicolumn{6}{|l|}{3 months } \\
\hline LVIDd (mm) & $46.9 \pm 1.1$ & $46.6 \pm 1.3$ & 0.96 & 58 & ${ }^{\mathrm{a}} 0.341^{\mathrm{ns}}$ \\
\hline LIVDs (mm) & $30.0 \pm 1.4$ & $30.5 \pm 1.6$ & 1.29 & 58 & ${ }^{\mathrm{a}} 0.198^{\mathrm{ns}}$ \\
\hline $\operatorname{LVEF}(\%)$ & $62 \pm 2$ & $61 \pm 2$ & 1.94 & 58 & ${ }^{\mathrm{a}} 0.058^{\mathrm{ns}}$ \\
\hline Wall motion abnormality & $1(3.3 \%)$ & $1(3.3 \%)$ & - & - & - \\
\hline
\end{tabular}

Group I Off Pump CABG In Left Main Coronary CABG patients between two groups. When chi Artery Disease. Group II On Pump CABG In Left Main Coronary Artery Disease. Chi Square test performed. Unpaired t test performed. $d f$ degree of freedom, Chi square test, p>0.05. Following table shows the post-operative NYHA changes among square test is performed there is no statistically significant ( $p>.05)$ changes between groups observed in 1st month, 2nd month and third month respectively.

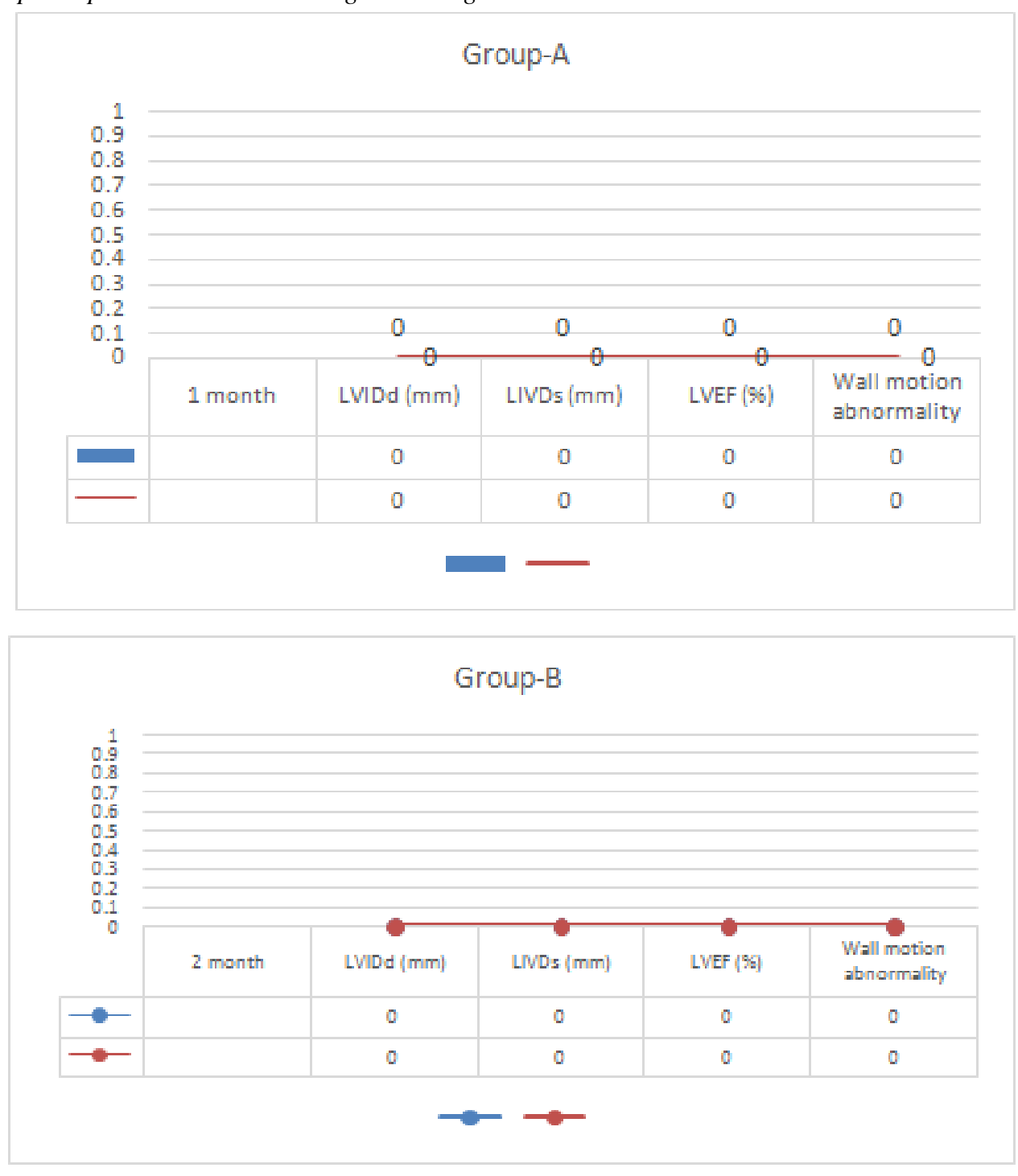


Post-Operative Echocardiographic Changes between two Groups of CABG Patients: A Study in the National Institute of Cardiovascular Disease (NICVD)

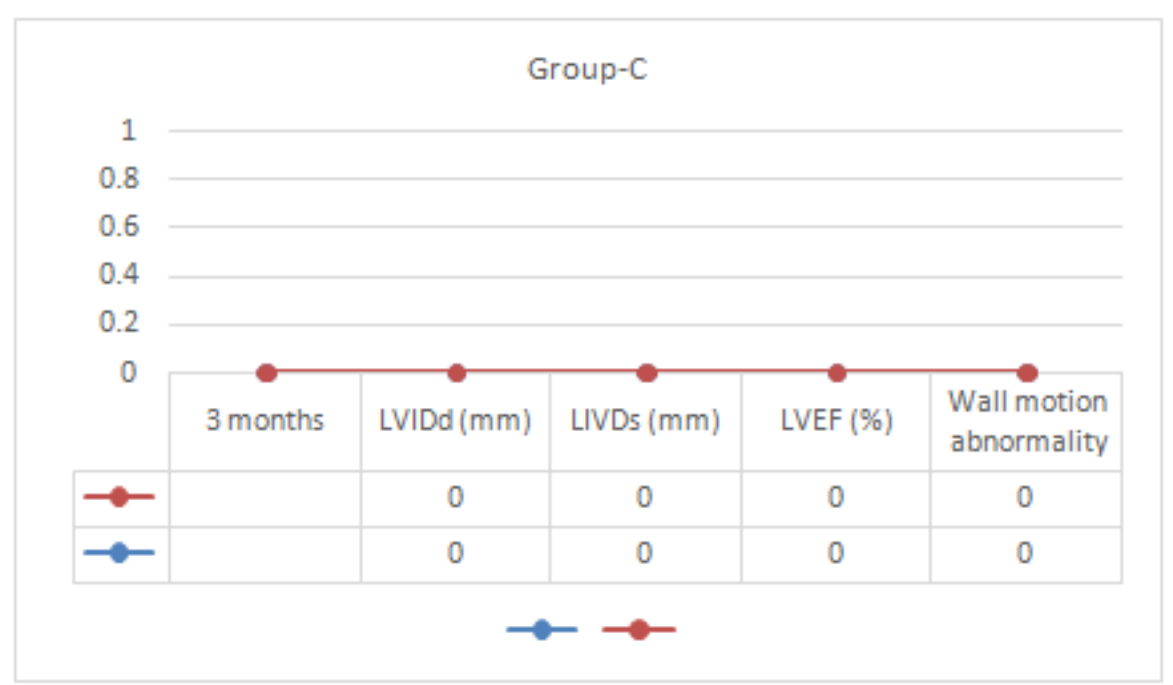

Figure2. Changes in Echocardigraphic variable between three groups $A, B, C$.

Table3. Change in NYHA functional Class post operatively

\begin{tabular}{|l|l|l|l|l|l|l|l|}
\hline \multicolumn{1}{|c|}{ NYHA Class } & Group I (n=28) n & \% & $\begin{array}{c}\text { Group II (n=29) } \\
\text { n }\end{array}$ & \% & $\boldsymbol{x}^{\mathbf{2}}$ value & df & P Value \\
\hline month & & & & & & & \\
\hline Class I & 18 & 64.0 & 13 & 46.4 & & & \\
\hline Class II & 12 & 40.0 & 17 & 56.6 & 1.67 & 1 & $0.196^{\text {ns }}$ \\
\hline 2 months & & & & & & \\
\hline Class I & 18 & 64.0 & 14 & 46.7 & & & \\
\hline Class II & 12 & 40.0 & 16 & 53.3 & 1.07 & 1 & $0.300^{\text {ns }}$ \\
\hline 3 months & & & & & & \\
\hline Class I & 19 & 63.3 & 14 & 46.7 & & & \\
\hline Class II & 11 & 36.7 & 16 & 53.3 & 1.68 & 1 & $0.194^{\text {ns }}$ \\
\hline
\end{tabular}

Group I Off Pump CABG In Left Main Coronary Artery Disease. Group II On Pump CABG In Left

\section{DISCUSSION}

Since the beginning of Coronary artery bypass surgery in our National Institute of Cardiovascular Disease and Hospital, Dhaka, Bangladesh in 1979 our competent professors and surgeons had been engaged in performing the pivotal rule in the field of on pump coronary artery bypass surgery in this country. They however gradually acquired the confidence to start off pump coronary artery bypass surgery in the year 1997 with tremendous success and achievement in the era of new technology. With further development of new devices like suction stabilizer and their availability in our country they conquered the enhanced skill of performing off pump CABG in our institute. In all over the world surgeon and cardiologist were bewared about the risk of managing coronary artery disease with left main stem stenosis, because it possess additive risk for the patients performing any kind of intervention like CABG or PCI [13] This study was performed in NICVD, Dhaka, Bangladesh which included 60 patients of
Main Coronary Artery Disease. Chi Square test performed. ns not significant. S significant, $p>0.05$. coronary artery disease with left main stem stenosis. In group I age range from 40 to 70 years with a mean \pm SD of $51.5 \pm 7.3$ years. The majority of the patients are in age group 45-50 years. In group II the age ranged from 40 to 70 years with a mean \pm SD of $54.7 \pm 6$ years, majority of the patients are in 51-55 years. There is no significance difference in age among groups. Similar type several studies done by [11, 14] there was no significant different between two groups.

Regarding distribution of sex among patients, there were 25 male and 5 female in group I and 27 male and 3 female in group II. Chi square value reveals that there are no significance differences in between male and female. But female sex may be an independent predictor of early mortality and morbidity in the group of patients undergoing CABG surgery due to left main stenosis. Further study is needed to prove this [15] however it is quite possible that sometimes left main coronary artery disease present with concomitant carotid artery and 
lower limb artery stenosis. But here in our study only one patient was found with concomitant left carotid artery stenosis in group II and underwent carotid endarterectomy along with CABG and two patients out of which 1 in group I and 1 in group II found concomitant external iliac and common femoral artery stenosis.Preoperative angiographic data was evaluated among two groups of patients. All patients had significant left main coronary artery disease. Significant means $>50 \%$ stenosis in the luman. And all patients had multi vessel disease along with left main disease. Out of them LAD main trunk involvement in group I were 18 and in group II 17 patients, the branches of LAD D1 and $\mathrm{D} 2$ respectively also involves in two groups, LCX main trunk involvement were in group I and II 8 and 7 patients respectively and its branches OM1 and OM2 also involved, RCA involvement in two groups were 6 and 5 patients and PDA involvement were 4 and 3 patients respectively in between two groups. The involvements of disease in the coronary arteries were not statistically significant in both groups. Most of the stenosed vessels were graftable, but few had diffuse disease without any scope of placement of a graft. In pre-operative echocardiogram study there is mean value of LVIDd in group I is $47.5 \pm 1.1 \mathrm{~mm}$ and in group II $47.1 \pm 1.7 \mathrm{~mm}$, LVIDs in group $\mathrm{I}$ is $30.3 \pm$ $1.4 \mathrm{~mm}$ and in group II $31.1 \pm 1.8 \mathrm{~mm}$, LVEF in group I $59 \pm 2 \%$ and in group II $58 \pm 2 \%$ respectively. There is no statistically significant difference between groups. Wall motion abnormality also predicted by the Echo which shows only $6(20 \%)$ of patients in group I and $8(26.7 \%)$ of patients in group II patients posses it which is also statistically not significant. Postoperative NYHA changes observed in two groups. The is no significant differences between groups, but it is obvious that either technique is effective in reduction of NYHA status among coronary artery disease patients. Post-operative Echo cardigraphic changes and wall motion abnormality changes were observed for three successive months. There are also some changes observes between two groups, but statistical analysis could not revealed any significant differences between two groups. However preoperatively in group I mean value of LVIDd was $47.5 \pm 1.1 \mathrm{~mm}$ which reduces to $47.5 \pm 1.1 \mathrm{~mm}$ in $1 \mathrm{st}$ month, $47.2 \pm 1.2 \mathrm{~mm}$ in $2 \mathrm{nd}$ month and $46.9 \pm 1.1 \mathrm{~mm}$ in 3rd month, which is not significant. Also in case of LVIDs which mean value preoperatively was $30.3 \pm 1.4 \mathrm{~mm}$ and with no change to $30.3 \pm 1.4$ in 1 st month,
$30.1 \pm 1.3$ in 2 nd month and $30.3 \pm 1.4$ in 3rd month. Whereas pre-operavily in group II mean value of LVIDd was $47.1 \pm 1.7 \mathrm{~mm}$ with no changes to $47.1 \pm 1.7 \mathrm{~mm}$ in $1 \mathrm{st}$ month, $46.9 \pm 1.4 \mathrm{~mm}$ in $2 \mathrm{nd}$ month and $46.6 \pm 1.3 \mathrm{~mm}$ in 3rd month, which is also not significant. Same is the case of LVIDs, mean value preoperatively was $31.1 \pm 1.8 \mathrm{~mm}$ and with no change to $31.1 \pm 1.8$ in 1 st month, $30.8 \pm 1.7$ in 2 nd month and $30.5 \pm 1.6$ in 3rd month. One study conducted by [15] depicts improved EF with coronary bypass surgery. This correlates with improved LV function, thus, improvement in cardiac symptoms such as dyspnea, reduced exercise capacity, fatigue and weakness. Chronic LV dysfunction due to viable but hibernating myocardium in patients with severe multiple disease is relatively common. This explains the improvement in $78 \%$ of the study population. Here in our study pre-operative EF was in group I $59 \pm 2$ and in group II $58 \pm 2$. But in post-operative follow up this value changes to in group I $59 \pm 2$ and in group II $58 \pm 2$ in 1 st month, $60 \pm 2$ in group I and $59 \pm 2$ in group II in 2nd month, 62 \pm 2 in group I and $61 \pm 2$ in group II in 3rd month respectively. Pre-operative wall motion abnormality was observed in echocardiogram in group I 6 patients and in group II 8 patients. In first month 2 patients in group I and 3 patients in group II, in 2nd month 1 patient in group I and 2 patient in group II, in 3rd month I patient in group I and 1 patient in group was recorded.

\section{Summary AND CONCLUSION}

In our institution more than a decade has almost over since the start of OPCAB surgery with gradual success and integrity. However development of new technology and strategy for off pump bypass surgery eliminated few obstacles in the pathway of progress. Moreover our surgeon's confidence and skilled manipulation enhance the tremendous success regarding off pump technique which superseded the conventional technique in every day practice. This study is the pilot study and it never studied in any place before in Bangladesh. My effort in this study was to establish the safety and effectiveness of off pump coronary artery bypass surgery in left main coronary artery disease.

\section{REFERENCES}

[1] Eagle, KA, Edwards, FH, Guyton, RA, Davidoff, R, Ewy, GA, Gardner, TJ, et al. 2004, 'ACC/AHA 2004 guideline update for coronary 
artery bypass graft surgery: A report of the American College of Cardiology /Amer ican Heart Association task force on practice guidelines (committee to update the 1999 guidelines for coronary artery bypass graft surgery) developed in collaboration with the American association for thoracic surgery and the society of thoracic surgeons', [Online], (Updated January 2004), Available at: <http:/w ww.acc.org/clinical/guidelines/cabg/cabg.pdf $\% 3$ e, $\% 5$ baccessed 10 July $2007 \% 5$ d.

[2] Bojar, RM, 2005, Coronary artery disease in manual of perioperative care in adult cardiac surgery, 4th ed, Oxford, Blackwell publishing, pp. 3-11,

[3] Sellke, FW, and Ruel, M, 2005, 'Surgical treatment of coronary artery disease and its complications in Sabiston \& Spencer Surgery of the chest, $7^{\text {th }}$ ed, eds Sellke FW, Nido P.J., Swanson SJ, Philadelphia, Pennsylvania. Elsevier Saunders.Vol.2, Ch 82. Pp.1459-1490.

[4] Sellke, FW, DiMaio, JM, Caplan, LR, Ferguson, TB, Gardner, TJ, Hiratzka, LF, 2005, 'Comparing on pump and off pump coronary artery bypass grafting: American heart associateion council on cardiovascular surgery and anesthesia in collaboration with the interdisciplinary working group on quality of care and outcome research', Circulation, vol. 111, pp. 2858-2864.

[5] Cohn, WE and Rosinberg, A, 2005, 'Surgical treatment of coronary artery disease and its complications in Sabiston \& Spencer Surgery of the chest, 7th ed, eds Sellke FW, Nido PJ., Swanson SJ, Philadelphia, Pennsylvania. Elsevier Saunders. Vol.2, Ch 82.pp. 1459-1490.

[6] Mahmud, KN, Sarker, SK, Momin, SM, 2005, 'CABG on beating heart in NICVD: a cost effective and safer approach to coronary artery disease', BangHJ, vol. 20, no.2, pp. 52-54.

[7] Caracciolo, EA, Davis, KB, Spoko, G, Kaiser, GC, Corley, SD, Schaff, H, et al. 1995, 'Comparison of surgical and medical group with left main coronary artery disease: long term CASS experience*, Circulation vol. 91, pp. 2325-2334

[8] Dewey, TM, Magee, MJ, Edgerton, J R, Mathison, M, Tennison, D, Mack, MJ, 2001, 'Off pump bypass grafting is safe in patients with left main disease', Ann Thorac Surgery, vol. 72, pp. 788-792.

[9] Legare, JF, Alex, M, Buth, KJ, Sullivan, JA, 2005, 'Assessing the risk of waiting for coronary artery bypass graft surgery among patients with stenosis of the Isft main coronary artery', CMAJ, vol.173, no. 4, pp. 371-375.

[10] Izumi, Y, Magishi, K, Ishikawa, N, Kimura F, 2006, 'On pump beating heart coronary artery bypass grafting for acute myocardial infarction', Ann Thorac Surg, vol. 81, pp. 573-576.

[11] Lu, JC, Grayson, AD, Pullan, DM, 2005, 'On pump versus off pump surgical revascularizat ion for left main stem stenosis: risk adjusted outcomes', Ann Thorac Surg, vol. 80, pp. 136143.

[12] Kouchoukos, NT, 2003, 'Stenotic and atheros clerotic coronary artery disease' in Kirklin/ Barr att-Boys Cardiac Surgery, 3rd ed, eds Koucho ukos NT, Blackstone EH, Doty DB, Hanley FL, Karp RB, Philadelphia, Pennsyl vania, Elseviar Science, Vol. 1 Ch. 7. pp. 354-435.

[13] Jonsson, A, Hammar, N, Nordquist, T, Ivert, T, 2006, 'Left main coronary stenosis no longer a risk factor for early and late death after coronary artery bypass surgery: an experience covering three decades', Eur J Cardiothorac Surg, vol. 30, pp. 311-317.

[14] Saba, D, Ener, S, Bicer, M, Aytac, II, Senkaya, I, Ozkan, H, 2004, 'Off pump bypass grafting in patients with significant left main coronary artery stenosis', Heart Vessels, vol. 19, pp. 8-12.

[15] Sansano, OM, Lopez, G, Garcia, J, Manapat, A, Diaz, R, Barril, J 2003, 'Effects of revascularization on the ejection fraction of patients with severe systolic dysfunction', Phil Jlnternl Med, vol. 41, pp.111-113.

Citation: Kazi Zahidul Hoque, et al,. Post-Operative Echocardiographic Changes between two Groups of CABG Patients: A Study in the National Institute of Cardiovascular Disease (NICVD). ARC Journal of Cardiology. 2019; 5(2): 1-9. doi:dx.doi.org/ 10.20431/2455-5991.0502001.

Copyright: () 2019 Authors. This is an open-access article distributed under the terms of the Creative Commons Attribution License, which permits unrestricted use, distribution, and reproduction in any medium, provided the original author and source are credited. 